\title{
BMJ open Hollow spheroids beyond the invasive margin indicate the malignant potential of colorectal cancer
}

To cite: Tamura $\mathrm{K}$,

Yokoyama S, leda J, et al. Hollow spheroids beyond the invasive margin indicate the malignant potential of colorectal cancer. BMJ Open 2011;1:e000179.

doi:10.1136/

bmjopen-2011-000179

- Prepublication history and additional material for this paper are available online. To view these files please visit the journal online (http:// bmjopen.bmj.com).

Received 13 May 2011 Accepted 24 June 2011

This final article is available for use under the terms of the Creative Commons Attribution Non-Commercial 2.0 Licence; see http://bmjopen.bmj.com

${ }^{1}$ Second Department of Surgery, Wakayama Medical University, School of Medicine, Wakayama, Japan ${ }^{2}$ Department of Clinical Laboratory Medicine, Wakayama Medical University, School of Medicine, Wakayama, Japan ${ }^{3}$ Department of Immunology, Beckman Research Institute at City of Hope, Duarte, California, USA

\section{Correspondence to}

Shozo Yokoyama; yokoyama@wakayama-med. ac.jp

Koichi Tamura, ${ }^{1}$ Shozo Yokoyama, ${ }^{1}$ Junji leda, ${ }^{1}$ Katsunari Takifuji, ${ }^{1}$ Tsukasa Hotta, ${ }^{1}$ Kenji Matsuda, ${ }^{1}$ Yoshimasa Oku, ${ }^{1}$ Takashi Watanabe, ${ }^{1}$ Toru Nasu, ${ }^{1}$ Shigehisa Kiriyama, ${ }^{1}$ Naoyuki Yamamoto, ${ }^{1}$ Yasushi Nakamura, ${ }^{2}$ John E Shively, ${ }^{3}$ Hiroki Yamaue ${ }^{1}$

\section{ABSTRACT}

Objective: Tumour budding formed by histologically undifferentiated cancer cells beyond the border of the tumour margin is associated with lymph node metastasis. However, hollow tumour nests, a possible histologically advanced phenotype of tumour budding, have not been discussed. We examined whether hollow spheroids exist beyond the border of the invasive margin and are associated with metastasis and prognosis. Moreover, we suggest that carcinoembryonic antigen-related cell adhesion molecule 1 (CEACAM1) isoform balance is associated with hollow spheroid formation.

Methods: Immunohistochemical analyses with CEACAM1 and M30 as an apoptosis marker were performed to examine the importance of hollow spheroid CEACAM1 expression and central cell apoptosis in hollow spheroid formation. The correlations between the presence of hollow spheroids beyond the invasive margin and the clinicopathological characteristics of 314 patients with colorectal cancer were retrospectively evaluated. A 3D culture with colorectal cancer cells transfected with CEACAM1 CDNA or shRNA was used to determine whether CEACAM1 isoform balance controls colorectal hollow spheroid formation.

Results: Hollow spheroid formation accompanying central cell apoptosis was confirmed by M30 staining and serial section with CEACAM1 staining. Of the 314 patients, $96(30.4 \%)$ were classified as having hollow spheroids. The presence of hollow spheroids is an independent risk factor for metastases and shorter survival. In 3D culture, CEACAM1 isoform balance modulated hollow spheroid formation of colorectal cancer cells.

Conclusions: Hollow spheroid formation beyond the border of the tumour margin in colorectal cancer is more important than tumour budding for the prediction of malignant potential.

\section{INTRODUCTION}

An isolated cancer cell or a cancer cluster of fewer than five cancer cells was previously defined as a budding. ${ }^{1}$ The correlation

\section{ARTICLE SUMMARY}

\section{Article focus}

- We examine whether hollow spheroids formed by polarised luminal cells with an inner empty space exist beyond the tumour margin in colorectal cancer tissue, and whether the presence of hollow spheroids correlates with metastasis and poor prognosis.

- We investigate the role of CEACAM1 in hollow spheroid formation.

\section{Key messages}

- The presence of hollow spheroids beyond the invasive margin of colorectal cancer is an independent risk factor for metastases and shorter survival.

- CEACAM1 isoform balance can modulate hollow spheroid formation.

Strength and limitations of this study

- This is the first report on the existence and clinical implications of hollow spheroids beyond the invasive margin of colorectal cancer.

- A validation study is needed for address the implications of hollow spheroids for colorectal cancer malignancy.

between budding and metastasis and poor prognosis in colorectal cancer has been reported. ${ }^{1-5}$ It had been shown that tumour budding was associated with lymphatic invasion and lymph node metastasis in rectal cancer. ${ }^{1}$ It was also reported that tumour budding was an important predictive factor for recurrence and poor prognosis in patients with colorectal cancer. ${ }^{2}$ Moreover, it has been shown that tumour budding was significantly correlated with lymph node metastasis, local recurrence, distant metastasis and shorter survival in advanced colorectal cancers. ${ }^{4-8}$ However, the progression of tumour budding, whether an isolated cancer cell or a cluster of less than five cancer cells, has still to be elucidated. Some reports 
demonstrated that the histological morphogenesis of cancer cells can be replicated in 3D culture. ${ }^{9}{ }^{10}$ Accordingly, it is speculated that an isolated cancer cell progresses to form budding cells, next a spheroid, and then a hollow spheroid with a lumen. In clinical specimens, hollow spheroids are often regarded as a part of tube formation and a continuous main tumour. In the current study, we report that hollow spheroids formed by polarised luminal cells with an inner empty space exist beyond the tumour margin in colorectal cancer tissue. Moreover, we show that the presence of hollow spheroids is more strongly correlated than tumour budding with metastasis and poor prognosis.

Carcinoembryonic antigen-related cell adhesion molecule 1 (CEACAM1) plays an important role in this process, including lumen formation and inner cell apoptosis in breast cancer cells. ${ }^{9-12}$ However, the role of CEACAM1 in colorectal lumen formation in 3D culture has not been discussed. CEACAM1 is a type I transmembrane glycoprotein expressed in epithelial cells, and has three or four extracellular domains and either the long (71-73 amino acids, CEACAM1-L) or short (8-10 amino acids, CEACAM1-S) cytoplasmic domain isoform. CEACAM1, previously known as biliary glycoprotein I or CD66a, is a member of the carcinoembryonic antigen family and the immunoglobulin superfamily. ${ }^{13}$ CEACAM1 is down-regulated in malignant tissues derived from the colon, ${ }^{14}{ }^{15}$ liver, $^{16}$ breast ${ }^{17}$ and prostate, ${ }^{18}$ whereas high expression of CEACAM1 is associated with the progression of gastric cancer, ${ }^{19}$ malignant melanoma $^{20}$ and lung cancer. ${ }^{21}{ }^{22}$ In colorectal cancer, it is known that down-regulation of CEACAM1 occurs at the adenoma or early cancer stage, ${ }^{14}{ }^{15}$ and CEACAM1 is reexpressed in advanced colorectal cancers. ${ }^{23}{ }^{24}$ Recently, we showed that re-expressed CEACAM1, especially with long cytoplasmic domain dominance, in advanced colorectal carcinoma is associated with tumour metastasis and shorter survival. ${ }^{25}$ In the present study, we demonstrate that CEACAM1 isoform balance with long cytoplasmic domain dominance plays an important role in hollow spheroid formation in colorectal cancer.

\section{MATERIALS AND METHODS \\ Patients}

Three hundred and fourteen consecutive patients with colorectal cancer who underwent surgery between January 2002 and August 2005 in Wakayama Medical University Hospital (WMUH) were enrolled in the present study. Forty-six patients had 46 stage I tumours, 125 stage II, 87 stage III, and 56 stage IV based on the UICC TNM classification. The mean age of the patients was $64.9 \pm 8.3$ years, and there were 185 males and 129 females. The tumours were resected from either the colon $(n=175)$ or the rectum $(n=129)$. The follow-up period was 5 years. Patients with stage III and IV tumours received postoperative chemotherapy based on 5-fluorouracil, whereas most stage I and II patients received no chemotherapy.
Immunohistochemistry

Immunohistochemical staining was performed using paraffin-embedded tissue sections. In brief, sections were de-paraffinised and autoclave-treated at $121^{\circ} \mathrm{C}$ for $7 \mathrm{~min}$ in $0.1 \mathrm{M}$ citrate buffer $(\mathrm{pH} 6.5)$. Endogenous peroxidase activity was blocked by incubation in $10 \%$ hydrogen peroxide for $5 \mathrm{~min}$ at room temperature. After washing with Tris buffer solution and incubation with $5 \%$ bovine serum albumin, the sections were incubated with the primary monoclonal antibody to 29H2 (ab49510, diluted 1:100; Abcam, Cambridge, MA, USA), CEACAM1-L polyclonal antibody (diluted 1:1000; Roche Applied Science, Rotkreuz, Switzerland), CEACAM1-S polyclonal antibody (diluted 1:1000; Roche) or M30 cytoDEATH monoclonal antibody (diluted 1:50; Roche) at $4^{\circ} \mathrm{C}$ for $18 \mathrm{~h}$. M30 monoclonal antibody detects caspase-cleaved cytokeratin 18, which is specific to apoptotic cells. ${ }^{26} 27$ A biotinylated secondary antibody and peroxidaseconjugated streptavidin were each applied at room temperature for $90 \mathrm{~min}$. Finally, the sections were incubated in 3' 3-diaminobenzidine for $5 \mathrm{~min}$, followed by haematoxylin counterstaining and mounting. All specimens were blindly reviewed twice by four individuals including a pathologist (KT, JI, SY and YN). In case of difference, the specimen was viewed again using a multihead microscope and the disagreement resolved by consensus.

\section{Cells}

Two human colorectal cancer cell lines were used in the present study. LS174T and HT29 cells were purchased from the American Type Culture Collection (Manassas, Virginia, USA). LS174T and HT29 cells were maintained in Eagle's Minimum Essential Medium (EMEM; Wako, Tokyo, Japan) or McCoy's 5A (Gibco, Grand Island, New York, USA) medium containing $10 \%$ fetal bovine serum (FBS; Gibco), $100 \mathrm{U} / \mathrm{ml}$ of penicillin G, $100 \mu \mathrm{g} / \mathrm{ml}$ of streptomycin, and $0.25 \mu \mathrm{g} / \mathrm{ml}$ of amphotericin B (Gibco) at $37^{\circ} \mathrm{C}$ in a $5 \%$ humidified $\mathrm{CO}_{2}$ atmosphere.

\section{Transfection and RNA interference}

Transfection of plasmid coding CEACAM1-4L or $-4 \mathrm{~S}$ and short hairpin RNA (shRNA) plasmids targeted to CEACAM1 was performed as previously described. ${ }^{25}$ Briefly, CEACAM1-4L or -4S cDNA cloned into the $\mathrm{pH}-$ $\beta$ actin vector ${ }^{28}(0.8 \mu \mathrm{g})$ was mixed with $1 \mu \mathrm{l}$ of Lipofectamine 2000 (Invitrogen, Carlsbad, California, USA) in a final volume of $100 \mu \mathrm{l}$ of Opti-MEM medium, and added to LS174T and HT29 cells grown to 40\% confluence in 24-well plates. At $48 \mathrm{~h}$ after transfection, G418 (Roche, Basel, Switzerland) was used to isolate stably transfected cells. Short hairpin RNA plasmids targeted to CEACAM1 were synthesised by SABiosciences (Frederick, Maryland, USA) as follows: insert sequence CCA CAA ATG ACA CTG GAA TCT, and gga atc tca ttc gat gca tac (negative control). Each plasmid $(0.8 \mu \mathrm{g})$ was transfected in the same way as outlined above. The expression level of CEACAM1 was confirmed as previously described. ${ }^{25}$ 


\section{Matrigel culture}

Matrigel (200 $\mu$, BD Biosciences, Bedford, MA, USA) was plated on 24-well plates and allowed to solidify for $15 \mathrm{~min}$ at $37^{\circ} \mathrm{C}$. After the Matrigel solidified, $0.5 \mathrm{ml}$ of the cell suspension in the culture medium containing $2 \times 10^{4}$ cells $/ \mathrm{ml}$ was directly seeded into each well and incubated at $37^{\circ} \mathrm{C}$ in a $5 \%$ humidified $\mathrm{CO}_{2}$ atmosphere for $2 \mathrm{~h}$. After incubation, Matrigel $(200 \mu \mathrm{l}, \quad 50 \%$ concentration) was added to the upper layer and allowed to solidify for $15 \mathrm{~min}$ at $37^{\circ} \mathrm{C}$. Complete culture medium was then added, and the plates were incubated at $37^{\circ} \mathrm{C}$ in a $5 \%$ humidified $\mathrm{CO}_{2}$ atmosphere. During culture, cells were regularly monitored for cell growth and lumen formation, which was scored by the presence or absence of a lumen. Culture medium was changed every 3 days until day 15 .

\section{Statistical analysis}

The Student t test, the $\chi^{2}$ test or Fisher's exact test were used for statistical evaluation, and clinicopathological factors determined to be significant by the $\chi^{2}$ test were analysed by multivariate logistic regression, and an OR with 95\% CI was calculated for each factor. The Kaplan-Meier method was used to estimate postoperative survival and the log-rank test was used to determine statistical significance. A Cox proportional hazards model was used to assess the risk ratio under simultaneous contributions from several covariates. p Values of $<0.05$ were considered to be statistically significant. Statistical calculations were performed using StatView V5.0 software (SAS Institute).

\section{RESULTS}

Hollow spheroids beyond the invasive margin of colorectal cancer

To clarify that the colorectal cancer nest had a hollow spheroid structure and that CEACAM1 and central cell apoptosis were involved in hollow spheroid formation, we performed immunohistochemistry with CEACAM1 and M30. A field with more than one hollow spheroid beyond the invasive margin was regarded as positive under $\times 100$ magnification. Of the 314 paraffinembedded primary tumour tissue sections stained with H\&E, 96 sections $(30.6 \%)$ showed cancer nests with a spheroid hollow beyond the invasive border (figure 1). CEACAM1 and CEACAM1-L, but not CEACAM1-S, were expressed on the luminal inner surface and in the central apoptotic cells (figure 1B). The central cells in the lumen were identified as apoptotic cells as shown by M30 staining (figure 1B). To clarify that the cancer nest had a hollow spheroid structure, serial $2 \mu \mathrm{m}$ sections of paraffin-embedded tumour tissue were subjected to immunohistological examination with CEACAM1 (29H2). The serial colorectal cancer tissue sections revealed a hollow spheroid structure, and not a tube formation (figure 1C). Budding was counted under $\times 200$ magnification. A field with less than five budding foci was viewed as negative and a field with more than five was viewed as positive. ${ }^{3}$ A total of 131 sections $(41.7 \%)$ were positive for budding at the invasive margin.

Hollow spheroids and the malignant potential of colorectal cancer

The $\chi^{2}$ test or Fisher's test for lymph node metastasis revealed that the presence of hollow spheroids, budding, depth of tumour invasion, lymphatic permeation, venous permeation and age $>65$ were statistically significant (table 1). Multivariate logistic regression analysis for lymph node metastasis showed that the presence of hollow spheroids $(\mathrm{p}<0.001)$ and lymphatic permeation $(\mathrm{p}=0.001)$ were independent risk factors (table 2). The $\chi^{2}$ test or Fisher's test for distant metastasis revealed that the presence of hollow spheroids, budding, venous permeation, lymphatic permeation and depth of tumour invasion were statistically significant (table 1). The independent risk factors in multivariate logistic regression analysis for distant metastasis were hollow spheroids $(\mathrm{p}<0.001)$ and budding $(\mathrm{p}=0.005)$ (table 2$)$.

Depth of tumour invasion, lymphatic permeation, venous permeation, lymph node metastasis, distant metastasis, budding and hollow spheroids were of prognostic value for survival in univariate analyses (table 3). Multivariate analyses with the seven parameters which in univariate analyses were significant regarding prognosis, revealed that the presence of hollow spheroids $(p=0.001)$ and distant metastasis $(p<0.001)$ were significant independent prognostic factors (table 3 ). The Kaplan-Meier method used for overall survival analysis showed that hollow spheroids were significantly associated with shorter survival. The 5-year survival of the hollow spheroid group was $44.8 \%$ compared to $88.9 \%$ for the non-hollow spheroid group $(p<0.0001)$ (figure 2A). The 5-year survival of the budding group was $60.3 \%$ compared to $86.3 \%$ for the non-budding group $(\mathrm{p}<0.0001)$ (figure $2 \mathrm{~B})$.

\section{Association between CEACAM1 and hollow spheroid formation}

Hollow spheroid formation of CEACAM1-4L or -4S transfected HT29 cells

Since CEACAM1 is known to have two cytoplasmic domain isoforms, in order to elucidate the effect of CEACAM1 overexpression on colorectal cancer hollow spheroid formation in 3D culture, HT29 cells moderately expressing CEACAM1 were transfected with a plasmid encoding the CEACAM1-4L or $-4 \mathrm{~S}$ isoform. Stable CEACAM1-4L or -4S transfected HT29 cells had significantly elevated CEACAM1 mRNA and protein levels, which was confirmed by RT-PCR and western blot analyses, respectively, as previously described..$^{25}$ CEACAM1-4L transfected HT29 cells formed a lumen, whereas CEACAM1-4S transfected HT29 cells formed a solid sphere (figure 3A). HT29 cells overexpressing CEACAM1-4L exhibited significantly more lumen formation $(\mathrm{p}<0.05)$ (figure 3B). Conversely, CEACAM1$4 \mathrm{~S}$ transfected HT29 cells exhibited significantly less lumen formation $(\mathrm{p}<0.01)$ (figure $3 \mathrm{~B})$. 
A

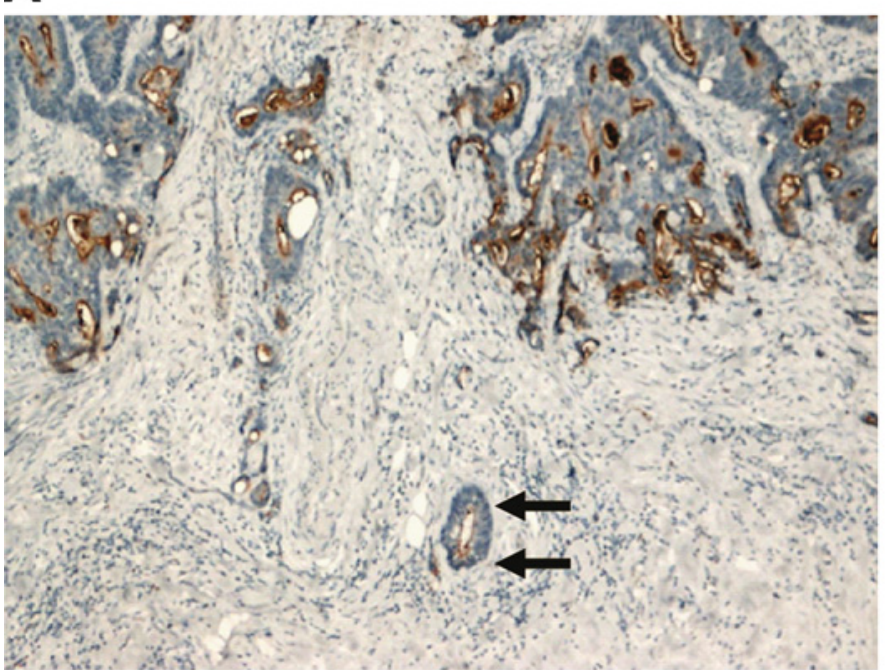

B

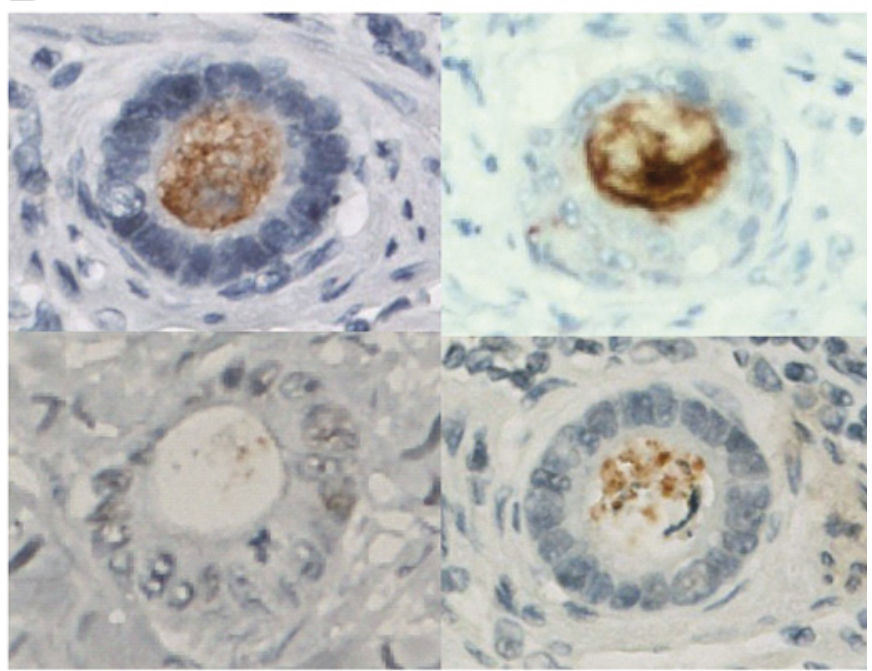

C

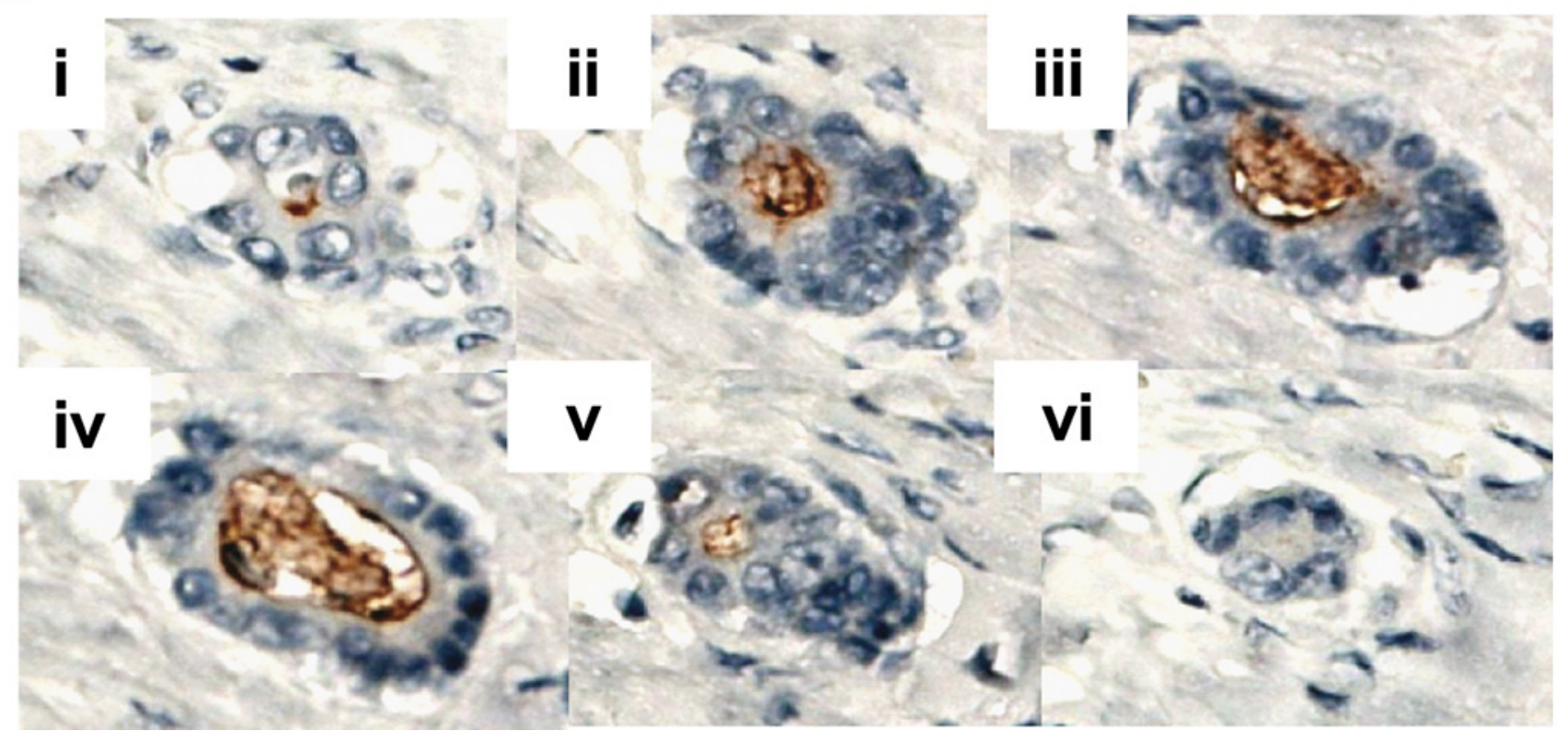

Figure 1 Immunohistochemical staining of hollow spheroids in colorectal cancer tissue. (A) Hollow spheroid, (arrows) beyond the invasive margin expressed CEACAM1 in the luminal surface $(29 \mathrm{H} 2)$ (original magnification, $\times 100)$. (B) The luminal surface and centre of the lumen were stained with CEACAM1 (left upper, CEACAM1 (29H2); original magnification, $\times 400)$, CEACAM1-L (right upper, CEACAM1-L specific antibodies; original magnification, $\times 400$ ), CEACAM1-S (left lower, CEACAM1-S specific antibodies, staining of endothelial cells and neutrophils were used as an internal positive control; original magnification, $\times 400)$ and M30 cytoDEATH (right lower; original magnification, $\times 400$ ). (C) Serial sections (i, ii, iii, iv, v, vi) from top (i) to bottom (vi) of a hollow spheroid stained with CEACAM1 $(29 \mathrm{H} 2)$. The lumen was a part of the hollow spheroid and was not a tube structure (original magnification, $\times 400)$.

Hollow spheroid formation of CEACAM1 shRNA transfected LS174T cells overexpressing CEACAM1-4L

To address whether CEACAM1 knock-down inhibits hollow spheroid formation by colorectal cancer cells, CEACAM1-4L overexpressing LS174T cells were transfected with shRNA against CEACAM1. Stable CEACAM1 shRNA transfected LS174T cells had significantly reduced CEACAM1 mRNA and protein levels, which was confirmed by RT-PCR and western blot analyses, respectively, as previously described. ${ }^{25}$ Vector control transfected LS174T cells formed a lumen, whereas CEACAM1
shRNA silencing LS174T cells formed a solid sphere (figure 3A). CEACAM1 knock-down cells exhibited significantly less lumen formation $(\mathrm{p}<0.01)$ (figure $3 \mathrm{C}$ ).

Hollow spheroid formation of CEACAM1-4S transfected LS174T cells overexpressing CEACAM1-4L

To determine whether disturbance of CEACAM1 cytoplasmic isoform balance affected colorectal cancer hollow spheroid formation, CEACAM1-4L overexpressing LS174T cells were transfected with plasmid encoding CEACAM1-4S. Stable CEACAM1-4S transfected LS174T 
Table 1 Univariate analyses of lymph node metastasis and distant metastasis

\begin{tabular}{|c|c|c|c|c|c|c|}
\hline & \multicolumn{3}{|c|}{ Lymph node metastasis } & \multicolumn{3}{|c|}{ Distant metastasis } \\
\hline & Present & Absent & p Value & Present & Absent & p Value \\
\hline Age & & & 0.017 & & & 0.475 \\
\hline$>65$ & 71 & 74 & & 30 & 139 & \\
\hline$<65$ & 60 & 109 & & 31 & 114 & \\
\hline Gender & & & 0.104 & & & 0.389 \\
\hline Male & 70 & 115 & & 39 & 146 & \\
\hline Female & 61 & 68 & & 22 & 107 & \\
\hline Tumour site & & & 0.564 & & & 0.084 \\
\hline Colon & 72 & 107 & & 41 & 138 & \\
\hline Rectum & 59 & 76 & & 20 & 115 & \\
\hline Histological type & & & 0.172 & & & 0.832 \\
\hline Moderate/well* & 125 & 180 & & 60 & 245 & \\
\hline Others $†$ & 6 & 3 & & 1 & 8 & \\
\hline Depth & & & $<0.001$ & & & $<0.001$ \\
\hline T3, 4 & 121 & 138 & & 61 & 198 & \\
\hline $\mathrm{T} 1,2$ & 10 & 45 & & 0 & 55 & \\
\hline Lymphatic permeation & & & $<0.001$ & & & $<0.001$ \\
\hline Present & 116 & 96 & & 56 & 156 & \\
\hline Absent & 15 & 87 & & 5 & 97 & \\
\hline Venous permeation & & & $<0.001$ & & & $<0.001$ \\
\hline Present & 106 & 105 & & 55 & 156 & \\
\hline Absent & 25 & 78 & & 6 & 97 & \\
\hline Budding & & & $<0.001$ & & & $<0.001$ \\
\hline Present & 78 & 53 & & 46 & 85 & \\
\hline Absent & 53 & 130 & & 15 & 168 & \\
\hline Hollow spheroids & & & $<0.001$ & & & $<0.001$ \\
\hline Present & 72 & 24 & & 45 & 51 & \\
\hline Absent & 59 & 159 & & 16 & 202 & \\
\hline
\end{tabular}

cells had significantly reduced CEACAM1-4L mRNA and protein levels, which was confirmed by RT-PCR and western blot analyses as previously described. ${ }^{25}$ The CEACAM1 and CEACAM1-4L protein levels of these cells were significantly suppressed compared to those of the parental or vector-transfected cells (control). ${ }^{25}$ CEACAM1-4S overexpressing cells reduced the number

Table 2 Multivariate analyses of lymph node metastasis and distant metastasis

\begin{tabular}{|c|c|c|c|}
\hline Variable & $\begin{array}{l}\text { No. of } \\
\text { patients }\end{array}$ & OR (95\% Cl) & p Value \\
\hline \multicolumn{4}{|l|}{$\begin{array}{l}\text { Lymph node } \\
\text { metastasis }\end{array}$} \\
\hline $\begin{array}{l}\text { Lymphatic } \\
\text { permeation }\end{array}$ & 212 & $\begin{array}{l}4.170 \\
(2.032 \text { to } 8.558)\end{array}$ & 0.001 \\
\hline Hollow spheroid & 96 & $\begin{array}{l}5.407 \\
(2.948 \text { to } 9.916)\end{array}$ & $<0.001$ \\
\hline \multicolumn{4}{|l|}{ Distant metastasis } \\
\hline Budding & 131 & $\begin{array}{l}2.840 \\
(1.381 \text { to } 5.837)\end{array}$ & 0.005 \\
\hline Hollow spheroid & 96 & $\begin{array}{l}6.334 \\
\text { (3.163 to } 12.683)\end{array}$ & $<0.001$ \\
\hline
\end{tabular}

of colorectal hollow spheroids formed $(\mathrm{p}<0.01)$ (figure 3D).

\section{DISCUSSION}

Most investigations for colorectal cancer have shown that tumour budding beyond the invasive margin is a risk factors for lymph node metastasis. ${ }^{12} 46^{-8}$ In the present study, hollow spheroids are more correlated with lymph node involvement, distant metastasis and overall survival than tumour budding, and multivariate analysis showed that the presence of hollow spheroids is an independent risk factor for metastasis and shorter survival. In particular, multivariate analysis of overall survival demonstrated that independent predictive factors for shorter survival are distant metastasis and hollow spheroids, not tumour budding, indicating that the presence of hollow spheroids is strongly predictive of the malignancy of colorectal cancer. We have previously shown that CEACAM1-L dominance in colorectal cancer is correlated with shorter survival. ${ }^{25}$ In the present study, we provided data showing that hollow spheroids expressed CEACAM1-L, not $-\mathrm{S}$, in the luminal surface, and that their formation is promoted by CEACAM1-4L expression. Kaplan-Meier analysis demonstrated that the hollow spheroid group had 
Table 3 Univariate and multivariate analyses of survival

\begin{tabular}{|c|c|c|c|c|}
\hline \multirow[b]{2}{*}{ Variable } & \multicolumn{2}{|l|}{ Univariate analysis } & \multicolumn{2}{|l|}{ Multivariate analysis } \\
\hline & HR (95\% Cl) & $\overline{p \text { Value }}$ & HR (95\% Cl) & p Value \\
\hline Age $(<65)$ & $1.228(0.785$ to 1.919$)$ & 0.368 & & \\
\hline Gender (male) & $0.974(0.618$ to 1.536$)$ & 0.911 & & \\
\hline Histologic type (others)* & $1.522(0.480$ to 4.828$)$ & 0.476 & & \\
\hline Depth (T3, T4) & 9.309 (2.285 to 37.920$)$ & 0.002 & $3.416(0.793$ to 14.727$)$ & 0.099 \\
\hline Lymphatic permeation & 3.307 (1.746 to 6.262$)$ & 0.001 & $1.024(0.498$ to 2.106$)$ & 0.948 \\
\hline Venous permeation & 2.200 (1.252 to 3.868$)$ & 0.006 & $0.954(0.529$ to 1.721$)$ & 0.876 \\
\hline Lymph node metastasis & $4.323(2.635$ to 7.093$)$ & $<0.001$ & $1.663(0.951$ to 2.909$)$ & 0.075 \\
\hline Distant metastasis & 9.126 (5.787 to 14.391$)$ & $<0.001$ & 3.799 (2.251 to 6.413$)$ & $<0.001$ \\
\hline Budding & 3.562 (2.209 to 5.743$)$ & $<0.001$ & $1.302(0.760$ to 2.230$)$ & 0.336 \\
\hline Hollow spheroid & 6.881 (4.240 to 11.166$)$ & $<0.001$ & 2.849 (1.632 to 4.975$)$ & 0.001 \\
\hline
\end{tabular}

shorter survival. These results suggested that CEACAM1$\mathrm{L}$ is involved in hollow spheroid formation leading to poor prognosis. A cohort validation study is needed to address the importance of hollow spheroids for colorectal cancer malignancy.

Tumour budding is defined as the presence of individual free cells or undifferentiated cancer cells. ${ }^{1}$ This histological structure is also termed 'sprouting', 'focal dedifferentiation' or 'budding'. ${ }^{16}$ However, it remains unclear whether tumour budding cells survive or undergo apoptosis. Based on 3D culture experiments that replicate the histological morphogenesis of cancer cells, ${ }^{9} 10$ it was speculated that surviving and static dedifferentiated cells proliferate and form spheroids, followed by central cell apoptosis. In the present study, we clarified the existence of hollow spheroids beyond the invasive margin of colorectal cancer, indicating that the same phenomenon of hollow spheroid formation in 3D culture may also occur in clinical colorectal cancer tissue. Further investigations are required to determine if hollow spheroids are derived from tumour budding, although hollow spheroids do seem to be one of the advanced forms of tumour budding.

The mRNA level of CEACAM1 was shown to be down-regulated in colorectal adenoma and early adenocarcinoma. ${ }^{14}{ }^{15}$ However, some reports have demonstrated that CEACAM1 protein expression is upregulated in advanced adenocarcinomas, ${ }^{23}{ }^{24}$ and that the intensity of CEACAM1 protein expression is correlated with TNM stage ${ }^{24}$ but is not significantly correlated with either overall survival or disease-free survival. ${ }^{23}$ Recently, we demonstrated that CEACAM1 was reexpressed at the invasive margin of advanced colorectal cancers, and that CEACAM1-L dominant expression was associated with lymph node involvement, distant metastasis and shorter survival. ${ }^{25}$ The isoform balance of CEACAM1 is crucial in the discussion of CEACAM1 function. In the current study, it was demonstrated that CEACAM1-L, not $-\mathrm{S}$, was expressed on the luminal surface of hollow spheroids. The result suggested that CEACAM1-L is involved in hollow spheroid formation leading to poor prognosis.

Our results showed that CEACAM1-L plays an important role in colorectal lumen formation, in contrast to some reports that breast cancer lumen formation is due to CEACAM1-S. ${ }^{10}{ }^{29}$ Previous breast cancer studies have already demonstrated the discrepancy that either long or short isoforms can promote lumen formation under different conditions. ${ }^{10} 122^{29}{ }^{30}$ In 3D culture experiments, CEACAM1-4S mediates lumen formation in MCF7 breast cancer cells, ${ }^{10}{ }^{29}$ whereas CEACAM1-4L induced lumen formation in a mouse model. ${ }^{12}{ }^{30}$
Figure 2 Kaplan-Meier plots of overall patient survival times. (A) The survival times of the hollow spheroid group and the non-hollow spheroid group were analysed. The 5-year survival of the hollow spheroid group was $44.8 \%$ compared to $88.9 \%$ for the nonhollow spheroid group $(p<0.0001)$. (B) The survival times of the budding group and the nonbudding group were analysed. The 5 -year survival of the budding group was $60.3 \%$ compared to $86.3 \%$ for the non-budding group $(p<0.0001)$.
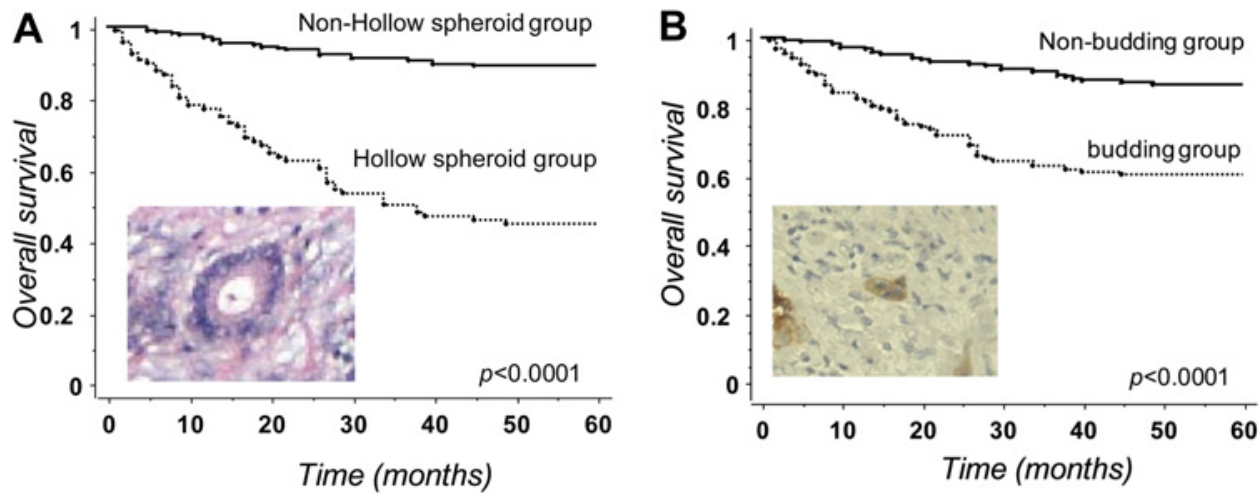
A
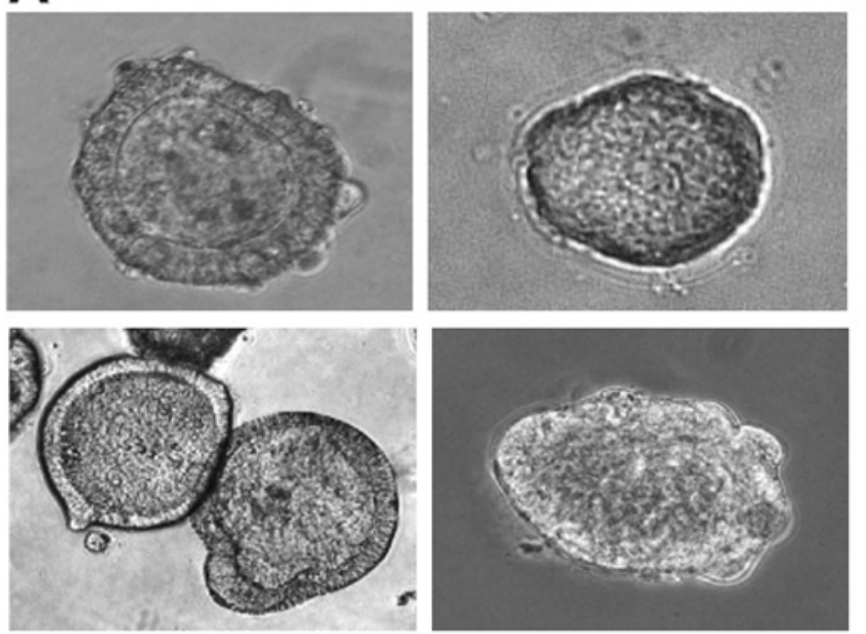

C

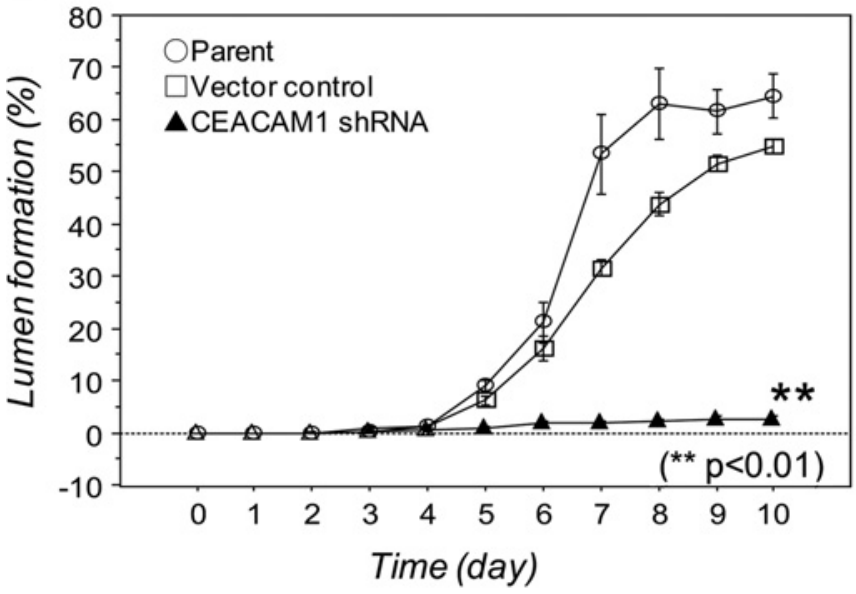

B

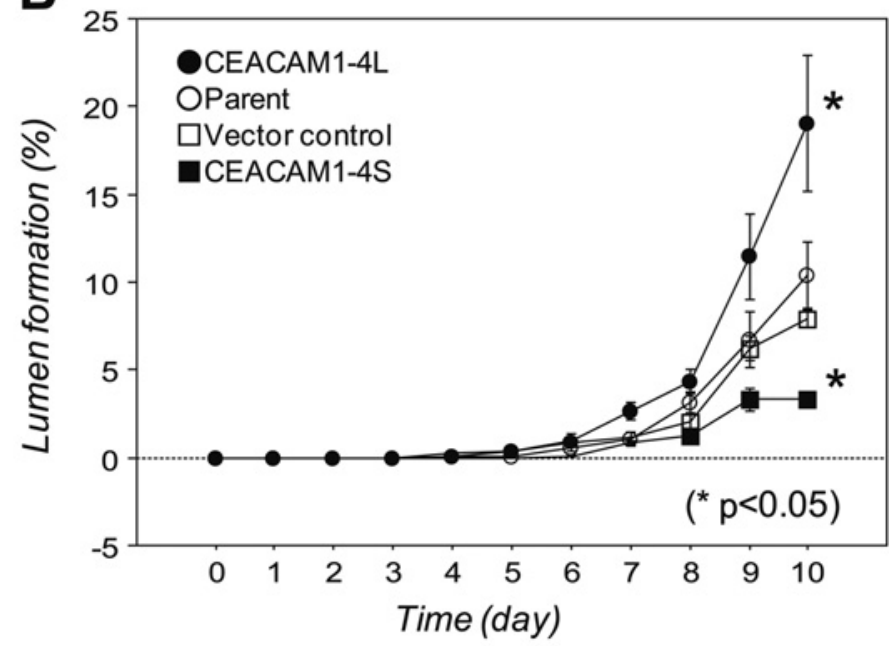

D

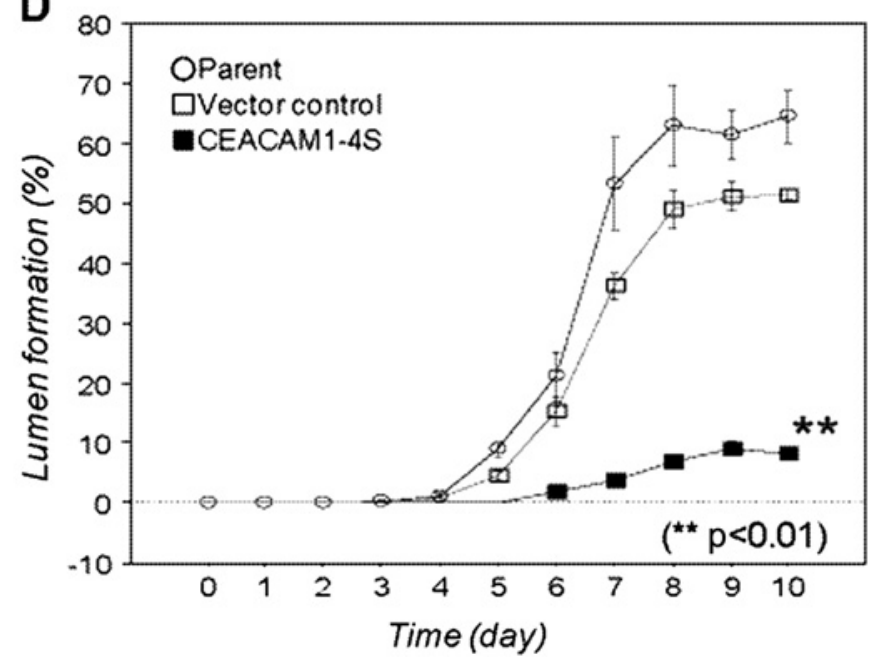

Figure 3 Morphological changes in colorectal cancer cells in 3D culture and time course of hollow spheroid formation. (A) CEACAM1-4L transfected HT29 cells (left upper); CEACAM1-4S transfected HT29 cells (right upper); vector control transfected LS174T cells (left lower); CEACAM1-4S transfected LS174T cells (right lower). (B) CEACAM1-4L and CEACAM1-4S transfected HT29 cells. (C) CEACAM1 shRNA transfected LS174T cells. (D) CEACAM1-4S transfected LS174T cells. Long: CEACAM1-4L transfected cells; Parent: parent cells; Short: CEACAM1-4S transfected cells; shRNA: CEACAM1 shRNA transfected cells; Vector: vector control transfected cells.

According to mutation analysis of CEACAM1-4S cytoplasmic domain, phosphorylation of the cytoplasmic domain is crucial for lumen formation. ${ }^{122930}$ Therefore, both the long and short cytoplasmic domain isoforms of CEACAM1, according to phosphorylation status, can induce lumen formation. The molecular mechanism of CEACAM1 in lumen formation remain to be investigated in greater detail.

Previous studies have shown that enforced overexpression of CEACAM1 in normal ${ }^{9}$ and cancerous ${ }^{10}$ breast cells induced inner cell apoptosis and hollow spheroid formation in 3D culture and a mouse model. ${ }^{12}$ CEACAM1-4S can mediate the reversion of breast cells from a malignant to a non-malignant morphology with a well differentiated structure in $3 \mathrm{D}$ culture ${ }^{10}{ }^{2}$ However, the association between hollow spheroids in colorectal cancer and CEACAM1 remains to be elucidated. Our clinical investigation showed that the luminal surface of hollow spheroids was stained with CEACAM1-L, but not the short isoform, and central cell apoptosis of hollow spheroids was shown by M30 (cleaved cytokeratin 18) staining. This finding, that hollow spheroids in colorectal cancer are formed by central cell apoptosis, is consistent with the breast cancer 3D culture model. Our 3D culture experiments showed that enforced CEACAM1-4L expression of colorectal cancer cells promoted hollow spheroid formation in 3D culture, and that hollow spheroid formation was suppressed by shRNA targeted to CEACAM1. These results indicated that CEACAM1 expression is important for hollow spheroid formation in colorectal cancer. We also showed that hollow spheroid formation was inhibited by overexpression of CEACAM1$4 \mathrm{~S}$, indicating that CEACAM1 isoform balance plays an important role in hollow spheroid formation.

CEACAM1-L contains two tyrosines in immunoreceptor-tyrosine-based inhibitory motifs (ITIMs) that can 
be phosphorylated by Src kinases, ${ }^{31}$ and once phosphorylated, can bind the SHP-1 and SHP-2 phosphatases. ${ }^{32}$ Two cytoplasmic tyrosines of CEACAM1-L affect in vivo tumour growth-inhibitory properties and intercellular adhesion. ${ }^{33}$ It has been shown that CEACAM1-L is able to bind actin and tropomyosin, and that CEACAM1-S can also bind actin, tropomyosin, annexin II and calmodulin. ${ }^{34}$ In normal colonic epithelial cells, CEACAM1-L constitutes $15 \%-20 \%$ of the total CEACAM1 protein. ${ }^{35}$ A significant overexpression of the CEACAM1-L isoform or reversion of the normal ratio of CEACAM1-L/-S isoforms reverses the tumour inhibition phenotype, whereas CEACAM1-L exhibits tumour inhibitory potential when the CEACAM1 isoforms are balanced normally. ${ }^{35}$ We herein demonstrated that CEACAM1-4L promoted hollow spheroid formation in colorectal cancer, while CEACAM1-4S inhibited hollow spheroid formation. The difference may be explained by the different functions of the long and short cytoplasmic domains.

Cytoplasmic domain splicing isoform balance affects the function of CEACAM1. The balance of CEACAM1 isoform expression has been evaluated in epithelial cells. ${ }^{35-37}$ Both long and short CEACAM1 cytoplasmic domain isoforms coexist in most normal tissues. The long isoform is usually expressed in smaller amounts than the shorter isoform. ${ }^{35}$ In normal colonic epithelial cells, CEACAM1-L accounts for $15 \%-20 \%$ of total CEACAM1 expressed. ${ }^{35}$ The long isoform has a tumour inhibition phenotype when expressed at physiological concentrations and in the appropriate ratio relative to the short isoform. However, significant overexpression of the long isoform or disruption of the normal $\mathrm{L} / \mathrm{S}$ ratio abolished the tumour suppressive function. ${ }^{35}$ Therefore, adjustment of the relative ratio of the CEACAM1 isoforms may cause reversion to the normal phenotype. Although further investigation is required to clarify the appropriate isoform balance, CEACAM1 splicing control may be a therapeutic option.

It is important to discuss the function of the carcinoembryonic antigen (CEA) family in molecular and cellular biology. It was shown that only carcinoembryonic antigen-related cell adhesion molecule 6 (CEACAM6) expression, and not CEA or CEACAM1, is an independent prognostic factor for resectable colorectal cancer. $^{23}$ Therefore, CEACAM6 may also be involved in hollow spheroid formation. The association between hollow spheroid formation and other CEA family members such as CEACAM6 and CEA remains to be investigated.

It is speculated that hollow spheroids may form in glandular sites distant from the original lesion. Therefore, hollow spheroids may have acquired tumour-initiating properties. Tumour-initiating cells are essential for tumour growth in a distant lesion. CD133 is one of the promising tumour-initiating markers. It has been reported that the CD133-positive cells in colorectal cancer are tumour initiating and have high tumourigenic potential. ${ }^{38} 39$ Several reports have recently shown that expression of CD133 correlated with poor prognosis in colorectal cancer patients. ${ }^{40}{ }^{41}$ We hypothesised that hollow spheroids at the invasive margin of colorectal cancers express CD133 as a tumour-initiating marker, and behave as a tumour-initiating phenotype. In the immunohistochemical staining of our preliminary experiments, the staining pattern of CD133 expression resembled that of CEACAM1 expression, indicating that there may be a molecular association between CEACAM1 and CD133 (see supplementary data). CD133 expressing hollow spheroids were observed in all patients with hollow spheroids, suggesting that some of the CEACAM1-expressing hollow spheroids may have tumour-initiating ability. Although further investigation for other tumour-initiating cell markers is required to address the association between hollow spheroids and tumour initiation, hollow spheroids beyond the invasion margin may be predictive of potent tumour-initiating growth at metastatic sites.

In conclusion, the present study demonstrated that hollow spheroids existed beyond the invasive margin and were an independent risk factor for lymph node involvement, distant metastases and shorter survival in patients with colorectal cancer. We also showed that CEACAM1 isoform balance plays an important role in central cell apoptosis in hollow spheroids. Identification of hollow spheroids beyond the invasive margin is important for recognition of the malignant potential of colorectal cancer.

Acknowledgements The authors thank Dr Brian Quinn, Editor-in-Chief, Japan Medical Communication for writing assistance.

Funding This study was supported by Grant-in-Aid 20591554 from the Ministry of Education, Culture, Sports, Science and Technology, Japan and by the Takeda Science Foundation, Japan.

Competing interests None.

Ethics approval The study was approved by the Human Ethics Review Committee of Wakayama Medical University (\#802).

Contributors KT performed immunohistochemistry, conducted cellular and molecular experiments, and carried out statistical analysis. SY conceived and designed the study. JI, SK and TN performed immunohistochemistry. KT, TH and KM carried out surgery. YO, NY and TW performed statistical analysis. YN confirmed the results of immunohistochemical analysis. JS helped in the conception and design of the study and in the production of antibodies. HY helped in the conception and design of the study. All authors drafted and critically revised the manuscript.

Provenance and peer review Not commissioned; externally peer reviewed.

Data sharing statement Immunohistochemistry and 3D culture data will be shared.

\section{REFERENCES}

1. Morodomi $\mathrm{T}$, Isomoto $\mathrm{H}$, Shirouzu $\mathrm{K}$, et al. An index for estimating the probability of lymph node metastasis in rectal cancers. Lymph node metastasis and the histopathology of actively invasive regions of cancer. Cancer 1989;63:539-43.

2. Hase K, Shatney C, Johnson D, et al. Prognostic value of tumor "budding" in patients with colorectal cancer. Dis Colon Rectum 1993;36:627-35.

3. Ueno $\mathrm{H}$, Mochizuki $\mathrm{H}$, Shinto $\mathrm{E}$, et al. Histologic indices in biopsy specimens for estimating the probability of extended local spread in patients with rectal carcinoma. Cancer 2002;94:2882-91. 
4. Ueno H, Murphy J, Jass JR, et al. Tumour 'budding' as an index to estimate the potential of aggressiveness in rectal cancer. Histopathology 2002;40:127-32.

5. Ueno $\mathrm{H}$, Mochizuki $\mathrm{H}$, Hashiguchi $\mathrm{Y}$, et al. Risk factors for an adverse outcome in early invasive colorectal carcinoma. Gastroenterology 2004:127:385-94.

6. Ono M, Sakamoto M, Ino $\mathrm{Y}$, et al. Cancer cell morphology at the invasive front and expression of cell adhesion-related carbohydrate in the primary lesion of patients with colorectal carcinoma with liver metastasis. Cancer 1996;78:1179-86.

7. Goldstein NS, Hart J. Histologic features associated with lymph node metastasis in stage T1 and superficial T2 rectal adenocarcinomas in abdominoperineal resection specimens. Identifying a subset of patients for whom treatment with adjuvant therapy or completion abdominoperineal resection should be considered after local excision. Am J Clin Pathol 1999;111:51-8.

8. Okuyama T, Oya M, Ishikawa $\mathrm{H}$. Budding as a risk factor for lymph node metastasis in pT1 or pT2 well-differentiated colorectal adenocarcinoma. Dis Colon Rectum 2002;45:628-34.

9. Huang J, Hardy JD, Sun Y, et al. Essential role of biliary glycoprotein (CD66a) in morphogenesis of the human mammary epithelial cell line MCF10F. J Cell Sci 1999;112(Pt 23):4193-205.

10. Kirshner J, Chen CJ, Liu P, et al. CEACAM1-4S, a cell-cell adhesion molecule, mediates apoptosis and reverts mammary carcinoma cells to a normal morphogenic phenotype in a 3D culture. Proc Natl Acad Sci U S A 2003;100:521-6.

11. Huang J, Simpson JF, Glackin C, et al. Expression of biliary glycoprotein (CD66a) in normal and malignant breast epithelial cells. Anticancer Res 1998;18:3203-12.

12. Yokoyama S, Chen CJ, Nguyen T, et al. Role of CEACAM1 isoforms in an in vivo model of mammary morphogenesis: mutational analysis of the cytoplasmic domain of CEACAM1-4S reveals key residues involved in lumen formation. Oncogene 2007;26:7637-46.

13. Thompson JA, Grunert F, Zimmermann W. Carcinoembryonic antigen gene family: molecular biology and clinical perspectives. J Clin Lab Anal 1991:5:344-66.

14. Neumaier M, Paululat S, Chan A, et al. Biliary glycoprotein, a potential human cell adhesion molecule, is down-regulated in colorectal carcinomas. Proc Natl Acad Sci U S A 1993;90:10744-8.

15. Nollau $\mathrm{P}$, Scheller H, Kona-Horstmann M, et al. Expression of CD66a (human C-CAM) and other members of the carcinoembryonic antigen gene family of adhesion molecules in human colorectal adenomas. Cancer Res 1997;57:2354-7.

16. Tanaka $\mathrm{K}$, Hinoda $\mathrm{Y}$, Takahashi $\mathrm{H}$, et al. Decreased expression of biliary glycoprotein in hepatocellular carcinomas. Int $J$ Cancer 1997;74:15-19.

17. Riethdorf L, Lisboa BW, Henkel U, et al. Differential expression of CD66a (BGP), a cell adhesion molecule of the carcinoembryonic antigen family, in benign, premalignant, and malignant lesions of the human mammary gland. J Histochem Cytochem 1997;45:957-63.

18. Luo W, Tapolsky M, Earley K, et al. Tumor-suppressive activity of CD66a in prostate cancer. Cancer Gene Ther 1999;6:313-21.

19. Kinugasa $\mathrm{T}$, Kuroki $\mathrm{M}$, Takeo $\mathrm{H}$, et al. Expression of four CEA family antigens (CEA, NCA, BGP and CGM2) in normal and cancerous gastric epithelial cells: up-regulation of BGP and CGM2 in carcinomas. Int J Cancer 1998;76:148-53.

20. Thies A, Moll I, Berger J, et al. CEACAM1 expression in cutaneous malignant melanoma predicts the development of metastatic disease. $J$ Clin Oncol 2002;20:2530-6.

21. Laack $E$, Nikbakht $H$, Peters $A$, et al. Expression of CEACAM1 in adenocarcinoma of the lung: a factor of independent prognostic significance. J Clin Oncol 2002;20:4279-84.

22. Sienel W, Dango S, Woelfle U, et al. Elevated expression of carcinoembryonic antigen-related cell adhesion molecule 1 promotes progression of non-small cell lung cancer. Clin Cancer Res 2003;9:2260-6.
23. Jantscheff $\mathrm{P}$, Terracciano L, Lowy $\mathrm{A}$, et al. Expression of CEACAM6 in resectable colorectal cancer: a factor of independent prognostic significance. J Clin Oncol 2003;21:3638-46.

24. Kang WY, Chen WT, Wu MT, et al. The expression of CD66a and possible roles in colorectal adenoma and adenocarcinoma. Int $J$ Colorectal Dis 2007;22:869-74.

25. leda J, Yokoyama S, Tamura K, et al. Re-expression of CEACAM1 long cytoplasmic domain isoform is associated with invasion and migration of colorectal cancer. Int J Cancer. Published Online First: 16 Mar 2011. doi:10.1002/ijc.26072.

26. Leers MP, Kolgen W, Bjorklund V, et al. Immunocytochemical detection and mapping of a cytokeratin 18 neo-epitope exposed during early apoptosis. J Pathol 1999;187:567-72.

27. Caulin C, Salvesen GS, Oshima RG. Caspase cleavage of keratin 18 and reorganization of intermediate filaments during epithelial cell apoptosis. J Cell Biol 1997;138:1379-94.

28. Gunning $\mathrm{P}$, Leavitt J, Muscat $\mathrm{G}$, et al. A human beta-actin expression vector system directs high-level accumulation of antisense transcripts. Proc Natl Acad Sci U S A 1987;84:4831-5

29. Chen CJ, Kirshner J, Sherman MA, et al. Mutation analysis of the short cytoplasmic domain of the cell-cell adhesion molecule CEACAM1 identifies residues that orchestrate actin binding and lumen formation. J Biol Chem 2007;282:5749-60.

30. Li C, Chen CJ, Shively JE. Mutational analysis of the cytoplasmic domain of CEACAM1-4L in humanized mammary glands reveals key residues involved in lumen formation: stimulation by Thr-457 and inhibition by Ser-461. Exp Cell Res 2009;315:1225-33.

31. Brummer J, Neumaier M, Gopfert C, et al. Association of pp60c-src with biliary glycoprotein (CD66a), an adhesion molecule of the carcinoembryonic antigen family downregulated in colorectal carcinomas. Oncogene 1995;11:1649-55.

32. Huber M, Izzi L, Grondin P, et al. The carboxyl-terminal region of biliary glycoprotein controls its tyrosine phosphorylation and association with protein-tyrosine phosphatases SHP-1 and SHP-2 in epithelial cells. J Biol Chem 1999;274:335-44.

33. Beauchemin N, Kunath $\mathrm{T}$, Robitaille $\mathrm{J}$, et al. Association of biliary glycoprotein with protein tyrosine phosphatase SHP-1 in malignant colon epithelial cells. Oncogene 1997;14:783-90.

34. Schumann D, Chen CJ, Kaplan B, et al. Carcinoembryonic antigen cell adhesion molecule 1 directly associates with cytoskeleton proteins actin and tropomyosin. $\mathrm{J}$ Biol Chem 2001;276:47421-33.

35. Turbide $\mathrm{C}$, Kunath $\mathrm{T}$, Daniels $\mathrm{E}$, et al. Optimal ratios of biliary glycoprotein isoforms required for inhibition of colonic tumor cell growth. Cancer Res 1997;57:2781-8.

36. Sundberg U, Obrink B. CEACAM1 isoforms with different cytoplasmic domains show different localization, organization and adhesive properties in polarized epithelial cells. J Cell Sci 2002;115 (Pt 6):1273-84.

37. Sundberg U, Beauchemin N, Obrink B. The cytoplasmic domain of CEACAM1-L controls its lateral localization and the organization of desmosomes in polarized epithelial cells. J Cell Sci 2004;117 (Pt 7):1091-104.

38. O'Brien CA, Pollett A, Gallinger S, et al. A human colon cancer cell capable of initiating tumour growth in immunodeficient mice. Nature 2007;445:106-10

39. Ricci-Vitiani L, Lombardi DG, Pilozzi E, et al. Identification and expansion of human colon-cancer-initiating cells. Nature 2007;445:111-15

40. Kojima M, Ishii G, Atsumi N, et al. Immunohistochemical detection of CD133 expression in colorectal cancer: a clinicopathological study. Cancer Sci 2008;99:1578-83.

41. Horst D, Kriegl L, Engel J, et al. CD133 expression is an independent prognostic marker for low survival in colorectal cancer. $\mathrm{Br} J$ Cancer 2008;99:1285-9. 